\title{
FAILURE MODE OF 2 FIBER POSTS BONDED USING 3 DIFFERENT ADHESIVE SYSTEMS
}

\author{
Adel Ahmed Siddiq*, Mohammad M Rayyan**, Lucette Segaan ${ }^{* * *}$, \\ Ehab A Farghaly ${ }^{* * * *}$ and Ghada E Hamza*****
}

\begin{abstract}
Purpose: evaluate the bond strength of 2 fiber post systems to root canal dentine using 3 different protocols of dual-cure resin cements.

Materials and methods: Fifty-four freshly extracted human mandibular premolars were decoronated and endodontically treated. Post spaces were prepared to a depth of $8 \mathrm{~mm}$ and width of $1.3 \mathrm{~mm}$, using manufacture's supplied drills. Teeth were randomly assigned into 2 groups $(n=27)$, according to fiber post light-transmission: group I; non-light transmitting fiber posts (Reforpost, Angelus; Brasil), group II; light transmitting fiber post (Exacto, Angelus; Brasil). Each group were further divided into 3 subgroups $(n=9)$ according to the adhesive protocol used (subgroup A; "SpeedCEM" self-adhesive, subgroup B; "Multilink Automix" self-etch, subgroup C; "Variolink II" total etch); "Ivoclar Vivadent; Liechtenstein". Using low speed saw (IsoMet, BUEHLER Worldwide Headquarters; USA), a $2 \mathrm{~mm}$ thickness coronal, middle and apical sections were obtained from each sample. All specimens were subjected to push out test using Instron machine (Lloyd Instruments Ltd; UK), until failure. Specimens were investigated under the stereomicroscope (BM1000; Jiangan Novle Optics Co., China) to study the mode of failure. Data was collected and statistically analyzed.
\end{abstract}

Results: In non-light transmitting group (GI); self-etch scored highest cohesive values (13), followed by self-adhesive (7) and finally total-etch (6). Regarding light transmitting group (GII); highest score was found in total-etch (11), followed by self-etch (8), and finally the self-adhesive(1).

Conclusions: Self-etch resin cement with a non-light transmitting fiber post might be a good choice to be used.

KEYWORDS: Self-etch resin cement with a non-light transmitting fiber post may be a good restorative choice for endodontically treated teeth.

* Clinical Instructor of Prosthodontics, Faculty of dentistry, Beirut Arab University, Beirut, Lebanon.

** Associate Professor of Prosthodontics, Beirut Arab University, Faculty of Dentistry, Assistant Professor of Fixed Prosthodontics, Faculty of Dentistry, Misr University for Science and Technology, Cairo, Egypt.

*** Professor of Prosthodontics, Faculty of dentistry, Beirut Arab University, Beirut, Lebanon.

**** Lecturer of Fixed Prosthodontics, Faculty of Dentistry, Misr University for Science and Technology, Cairo, Egypt.

***** Lecturer of Fixed and Removable Prosthodontics Department, Oral and Dental Research Division, National

Research Center, Cairo, Egypt. 


\section{INTRODUCTION}

Recently years, adhesively bonded joints have been used extensively for various applications inside patients mouth ${ }^{1,2}$. Their adhesive joints were reported to have a good behavior under fatigue loads, also enables minimally invasive restorative techniques, and result in less stress concentrations compared to alternative joining techniques ${ }^{3,4}$. However, debonding of dental restoration is one of the most problems to the restorative dentist. Therefore, there is a need for reliable models to predict performance of these adhesively bonded assemblies. Most clinical failures involving reconstructed teeth with fiber posts are due to cementation failure of the posts ${ }^{5,6}$.

Until a few years ago, most of resin adhesives were available in 3 application steps (total-etch) ${ }^{7,8}$. Nowadays, 3 categories of resin are used for luting fiber posts into the root canal, and they are mainly classified as total-etch, self-etch and self-adhesive resin cements.

Studies evaluating the efficiency of adhesively bonded dental materials to tooth structure, so-called bond strength studies, are frequently published in the dental literature ${ }^{9,10}$. Although, the relevance of such testing is still a matter of scientific, dispute and prediction of the clinical performance of a tested adhesive-restorative combination, on the basis of bond strength data is ambiguous. Manufacturers and researchers alike have a tendency to emphasize, without supporting evidence the results of such in vitro tests as determinants of bonding quality. They rely on strength test as a quality assurance test for bonding process ${ }^{11,12}$, also many researchers have even used such test for selection of their products and the appropriate surface preparation method.

However, the most important is to investigate the main cause of failure and its effect on the durability of the restoration. The failure to recognize the cause of such bond failures, has meant the continued use of deficient bonding processes, and has resulted in inappropriate test methods being used to assist selection of bonding materials and processes.

Unfortunately, in many studies failure patterns are recorded, yet not or only insufficiently discussed ${ }^{13}$. This test is one of the most important tests is also one of the most disparaged. Therefore the question arises, why failure analyses are performed unless they are qualified descriptors of the performance of the bonding. Leloup et al. ${ }^{7}$ found a significant positive correlation between the mean dentin bond strength and the rate of cohesive debonding failure from their review of 75 articles.

Debonding of adhesive joints occurs by a process of crack formation, propagation, subsequent joint failure, and cracks form at defects along the interface.

There are 3 possible mechanisms of failure of adhesive bonding; cohesive failure, adhesive failure, and mixed failure.

Cohesion bond failures result in fracture of the adhesive, and are characterized by the clear presence of adhesive material on the matching faces of both adherends. Also the adhesive surface typically appears rough, and may have a lighter color than the bulk adhesive material.

Adhesion failures occur at the interface between the adhesive and the adherend, with residual adhesive remaining at any location on one surface only and absence of adhesive on one of the bonding surfaces. The surface of the adhesive is smooth and often replicates surface features from the adherend. Adhesion failures exhibit low strength and may occur with no applied load if degradation of the interface is complete.

Mixed-mode failure which is least understood, where there is a combination of cohesion and 
adhesion failure within the same bond. This lack of understanding how an adhesive bond transitions from a strong bond which exhibits cohesion failure, to a weak bond which exhibits adhesion failure make it difficult to the investigator to be sure if the bond failure started with cohesive failure within the cement itself, or the cause started on the adhesive joint.

This study may analyze different patterns of failure modes between fiber post and tooth structure, in attempt to possibly improve bonding quality and performance. The null hypothesis of the study was that, no statistical difference would be found between failure modes of different bonding protocols.

\section{MATERIALS AND METHODS}

Fifty-four freshly extracted mandibular second premolars were collected, cleaned to remove adherent soft or hard tissues on the roots. The teeth were then sterilized in an autoclave at $121^{\circ} \mathrm{C}, 15$ psi for 40 minutes and stored in distilled water for $72 \mathrm{hrs}$. The crowns were amputated horizontally at cemento-enamel junction with water-cooled high speed round-end tapered carbide bur (Komet; Germany). Root canal treatment was performed using lateral condensation technique by guttapercha (META BIOMED; Korea) and eugenol-free root canal sealer (MICRO-MEGA; France).

A clear chemical cured acrylic resin (VertexDental; Netherlands) blocks were fabricated to facilitate easy manipulation and handling of roots.

Specimens were divided into 2 groups, according to the type of fiber post used; group I: non-light transmitting fiber post (GI) "Reforpost; Angelus, Brasil" and group II: light transmitting fiber post (GII) "Exacto; Angelus, Brasil", etch group were further divided into 3 sub groups ( $n=9)$, according to the adhesive protocol that was implemented (subgroup A; "SpeedCEM" self-adhesive, subgroup B; "Multilink Automix" self-etch, subgroup C; "Variolink II" total etch); "Ivoclar Vivadent; Liechtenstein".

Gates Glidden and Peeso-reamers "MANI, Belgium" were used to prepare the post space to depth of $8 \mathrm{~mm}$, followed by the corresponding post drill (1.3 $\mathrm{mm}$ in diameter). Fiber posts were tried for proper length, wiped with alcohol and dried using oil-free air. All fiber post surfaces were treated with Monobond Plus silane (Ivoclar Vivadent; Liechtenstein). Each post was then cemented according to manufacture instructions.

Using low speed IsoMet saw (BUEHLER Worldwide Headquarters; USA), 3 post/dentin sections (coronal, middle and apical) was obtained from each specimen (Fig 1), with a thickness of $2 \mathrm{~mm}$. Then a push-out test was performed using universal LRX-plus testing machine (Lloyd Instruments Ltd.; UK) at a cross-head speed of $0.5 \mathrm{~mm} / \mathrm{min}$.

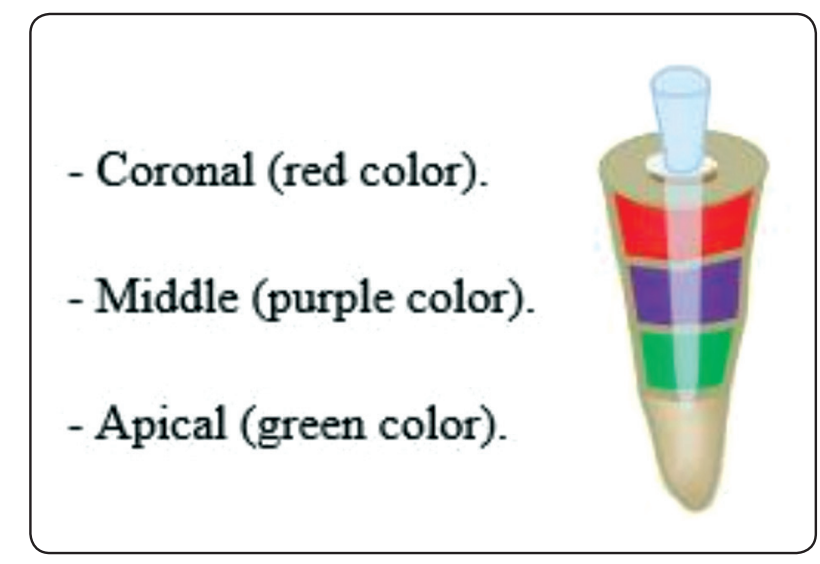

Fig. (1) Specimen sections

For each specimen, mode of failure was evaluated using Stereomicroscope (Fig. 2), and results were divided into 3 categories of failure (cohesive; within the cement, adhesive; between the cement and tooth surface, and mixed), then the data were collected, tabulated and statistically analyzed. 


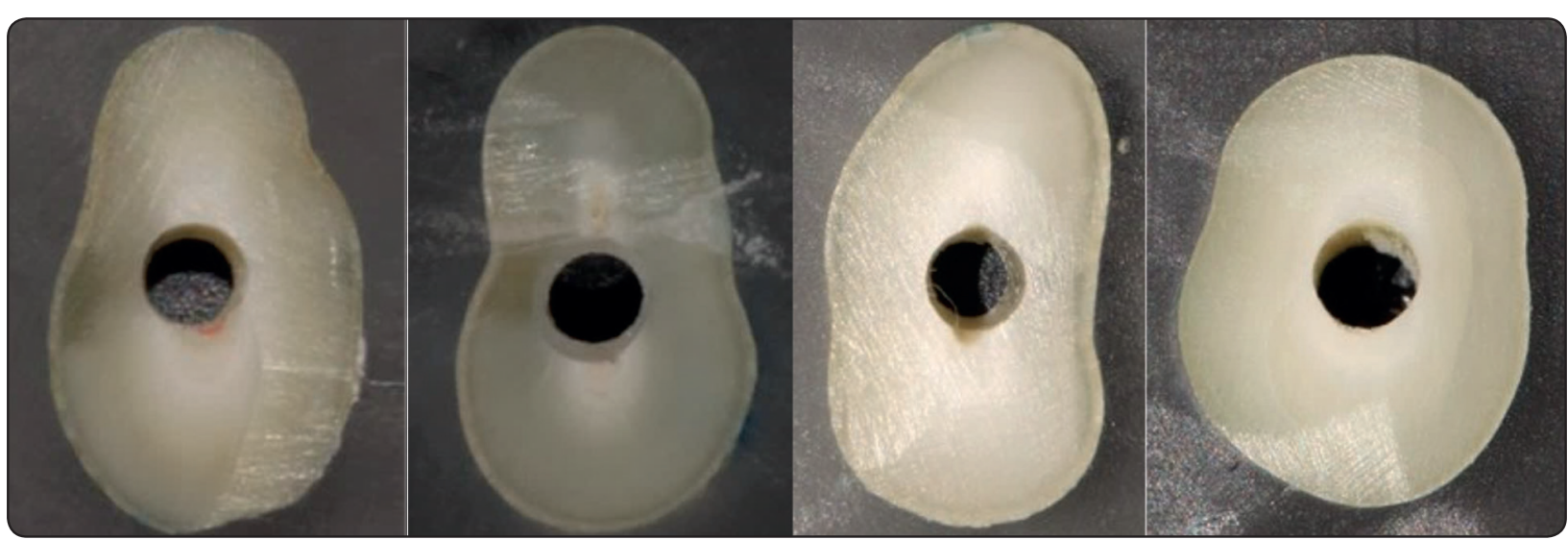

Fig. (2) sectional specimens after push-out test

\section{RESULTS}

After push-out test, the fracture mode of 162 specimens were evaluated, using Stereomicroscope at original magnification X40, the results of the added parts (coronal-middle-apical) of each subgroup are displayed in (table 1 \& Fig. 3,4):

\section{Comparing the subgroups to each other's the} results shown:

1- In non-light transmitting group (GI); the highest cohesive failure value was found on the selfetch subgroup which scored 13, followed by self-adhesive subgroup with a score of 7 and finally the total-etch subgroup which scored 6 . On the opposite side an adverse results were shown for the adhesive mode of failure.

2- In light transmitting group (GII); the highest cohesive mode of failure values was found on the total-etch subgroup with a score of 11, followed by self-etch subgroup with a score of
8 , and finally the self-adhesive subgroup which scored 1.

3- Comparing the non-light and light subgroup; a better values on the cohesive mode of failure on both self-etch and self-adhesive subgroups were scored under the light transmitting group. However the highest value of total etch subgroup was on non-light transmitting group.

\section{Comparing the non-light (GI) and light (GII) transmitting groups:}

- As shown in (Fig: 5,6); the results showed a high difference on the cohesive mode of failure percentage, which scored $26 \%$ in GI and $4 \%$ in GII. On the other hand, adhesive failure scored $18 \%$ in GI and $41 \%$ in GII.

- The mixed mode of failure almost scored a near percentage in both groups, which was $56 \%$ in GI, and $55 \%$ in GII.

TABLE (1) Fracture mode results for the added parts of non-light and light transmitting groups.

\begin{tabular}{|c|c|c|c|c|c|c|}
\hline & \multicolumn{3}{|c|}{ Non-light } & \multicolumn{3}{c|}{ Light } \\
\cline { 2 - 8 } & Self-adhesive & self-etch & total-etch & Self-adhesive & self-etch & total-etch \\
\hline Adhesion Failure & 5 & 3 & 6 & 11 & 6 & 5 \\
\hline Cohesion Failure & 7 & 13 & 6 & 1 & 8 & 11 \\
\hline Mixed Failure & 15 & 11 & 15 & 15 & 13 & 11 \\
\hline
\end{tabular}




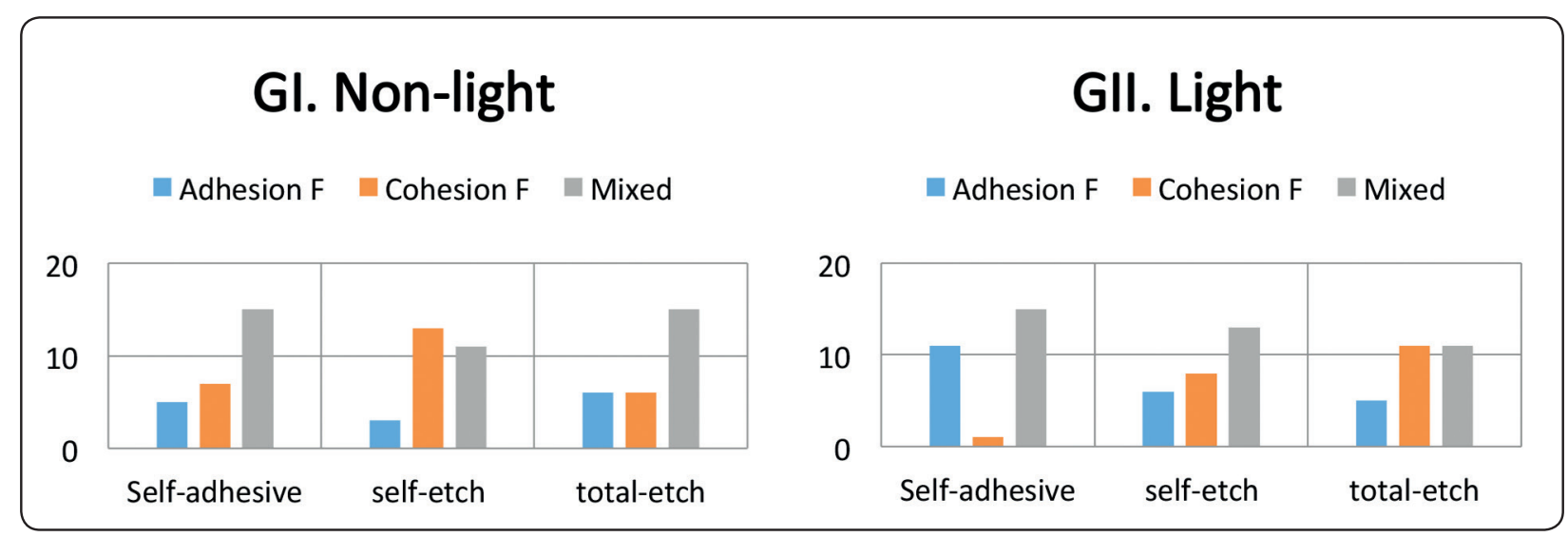

Fig. 3,4: Fracture mode charts for the added parts of non-light and light transmitting groups.

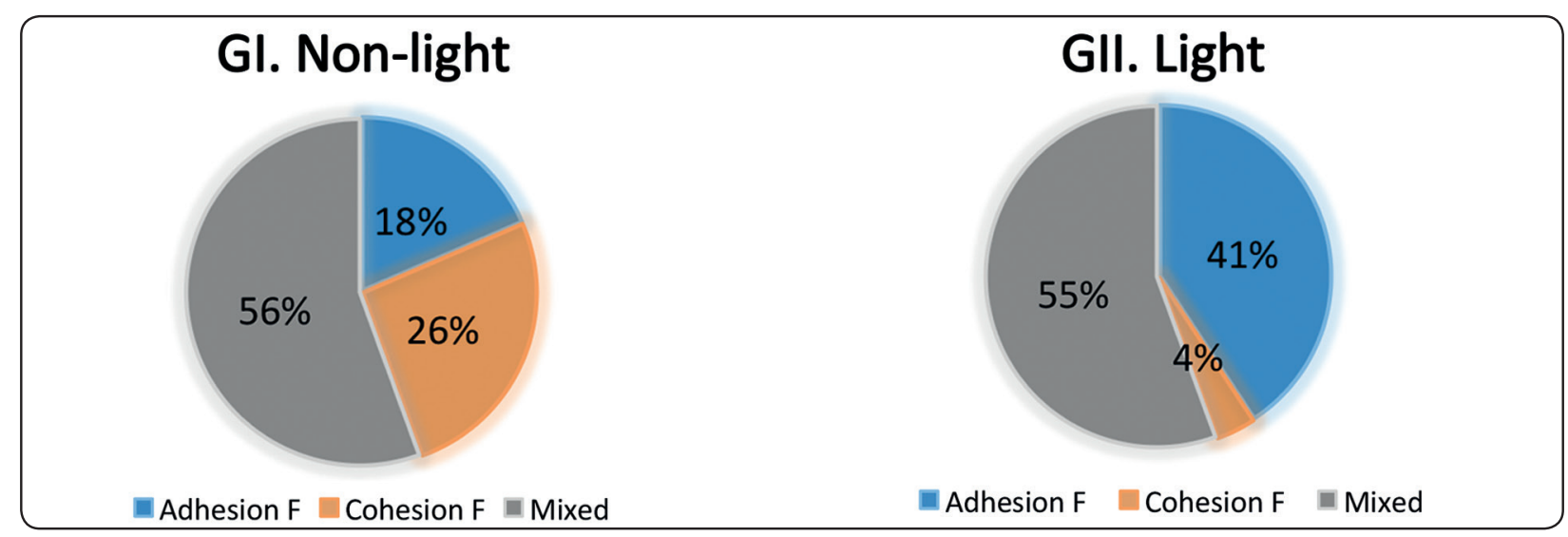

Fig. 5,6: Fracture mode charts for non-light and light transmitting groups.

\section{DISCUSSION}

For all practical purposes, the bulk properties of the tooth substrates (enamel and dentin) and restorative substrates are much stronger than the bond strength of the restorations. Therefore, cracks that form generally remain in the bonded interface zone. As cracks grow, they contribute to stress concentrations, or stress redistributions within the substrates. The final failure may often extend for short distances through portions of tooth structure or restorative material. Therefore failed surfaces should be examined carefully with moderate magnification to identify the origin of the critical crack.
In an ideal adhesive substrate system, the adhesive will fail cohesively. Cohesive failures are ideal in terms of surface preparation, because they indicate that the adhesive is the limiting variable in the equation. When this happens, it suggests that the adhesive had a stronger grip to the surface than itself, which shows a high degree of surface adhesion. Alternately, an adhesive failure occurs when the entire adhesive is preferentially left on one substrate and can be indicative of poor surface preparation.

Results of this study demonstrated a marked drawback of the cohesive values in both selfetch and self-adhesive subgroups, while using light transmitting fiber post, this might be due to 
the polymerization shrinkage of the resin. These findings are consistent with the existing literatures in 2014; Daphne, et al ${ }^{14}$, and Kansad, et al ${ }^{15}$, which showed that a self-etch system may create a better bond to the cervical, middle, and apical thirds of the radicular dentin than total-etch and self-adhesive cements.

Polymerization shrinkage may be the cause of inadequately bonded areas that may later lead to debonding. Further contraction of adhesive materials, during polymerization can cause gap formation between the restoration and the tooth structure. The adhesive may then pull away from the substrate, or stresses may be created that weaken the bond. Shrinkage is toward the center of the adhesive mass. However, shrinkage in light-cured systems occurs toward the light source. This polymerization shrinkage also when the adhesive shrinkage toward the source of light, this keeps the other surface of adherand under tension force and make it less reliable to fatigue stresses. Numerous authors 16,17 have reported that fiber post cementation with the resin cement, associated with etch-and-rinse adhesive and self-adhesive, may generate greater bonding potential than self-etch. In 2009, Ivana, et al ${ }^{18}$, concluded that, self-etching approach may offer less favourable adhesion to root canal dentin in comparison with etch-and-rinse and self-adhesive approaches. On the other hand, an adverse behavior was found on the total-etch subgroup, this might be due to the existed cured bond layer, which might balance the shrinkage effect of light by chemically bonded to the adhesive layer. The null hypothesis was rejected as there were a statistical difference between failure modes of different tested groups.

It should be highlighted that all in-vitro studies have limitations and cannot completely replace clinical trials. More studies should investigated the effect of light and the curing mode on the mode of failure and durability of dental adhesives.

\section{CONCLUSIONS}

\section{Within the limitations of this study it could be concluded that:}

1- Self-etch resin cement with a non-light transmitting fiber post, may offer a good restorative choice for endodontically treated teeth.

2- Light transmitting posts did not offer best bond when used with various adhesive systems.

\section{REFERENCES}

1- Rathke A, Haj-Omer D, Muche R (2009). Effectiveness of bonding fiber posts to root canals and composite core build-ups. Eur J Oral Sci. 117(5):604-10.

2- D'Arcangelo C, Zazzeroni S, D'Amario M (2008). Bond strength of three types of fiber-reinforced post systems in various regions of root canals. Int Endod J. 42: 322-328.

3- Bitter K, Priehn K, Martus P (2006). In vitro evaluation of push-out bond strengths of various luting agents to toothcolored posts. J Prosthet Dent. 95:302-310.

4- D'Arcangelo C, D'Amario M, Vadini M (2008). An evaluation of luting agent application technique effect on fiber post retention. J Dent. 36: 235-240.

5- Axelsson P, Lindhe J, Nystrom B. (1991). On the prevention of caries and periodontal disease. Results of a 15-year longitudinal study in adults. J Clin Periodontol. 18:182-189.

6- Testori T, Badino M, CastagnolaM. (1993). Vertical root fractures in endodontically treated teeth: a clinical survey of 36 cases. J Endod. 19:87-91.

7- De Munck J, Vargas M, Van Landuyt K (2004). Bonding of an auto-adhesive luting material to enamel and dentin. Dent Mater. 20:963-71.

8- Hikita K, Van Meerbeek B, De Munck J (2007). Bonding effectiveness of adhesive luting agents to enamel and dentin. Dent Mater. 23:71-80.

9- Goracci C, Sadek FT, Fabianelli A (2005). Evaluation of the adhesion of fiber posts to intraradicular dentin. Oper Dent. 30(5):627-35.

10- Amaral M, Rippe MP, Bergoli CD (2011). Multistep adhesive cementation versus one-step adhesive cementation: push-out bond strength between fiber post and root dentin before and after mechanical cycling. Gen Dent. 59(5):e185-91 
11- Goracci C, Tavares AU, Fabianelli A (2004). The adhesion between fiber posts and root canal walls: comparison between microtensile and push-out bond strength measurements. Eur J Oral Sci. 112(4):353-61.

12- Soares CJ, Santana FR, Castro CG (2008). Finite element analysis and bond strength of a glass post to intraradicular dentin: comparison between microtensile and push-out tests. Dent Mater. 24(10):1405-11.

13- Othman HI, Elshinawy MI, Abdelaziz KM. (2013). Retention of fiber posts to the optimally and over-prepared dowel spaces. J Adv Prosthodont. 5(1):16-20.

14- Daphne Câmara Barcellos, Maria Filomena Rocha Lima Huhtala (2014). Influence of adhesive system in bond strength of fiber glass posts to radicular dentin using dual cure resin cement. Braz Dent Sci. 17(1).
15- Kansad Pala, Sezer Demirbuga (2014). Effect of different bonding techniques on the bond strength of two different fiber. J Res Dent. 2:32-6.

16- Parnian Alizadeh Oskoee, Elmira Jafari Navimipour (2011). Effect of Different adhesion strategies bond strength of fiber reinforced composite posts. African journal of biotechnology. 10(76):17593-98.

17- Mazzoni A, Marchesi G, Cadenaro M (2009). Pushout stress for fiber posts luted using different adhesive strategies. Eur J Oral Sci. 117(4):447-53.

18- Ivana Radovic, Claudia Mazzitelli, Nicoletta Chieffi, Marco Ferrari. (2009). Evaluation of the adhesion f fiber posts cemented using different adhesive approaches. European Journal of Oral Sciences. 116(6):557-63. 